\title{
Timing and spectral changes of the Be X-ray transient EXO 0531-6609.2 through high and low state
}

\author{
N. Rea ${ }^{1,2}$, G. L. Israel ${ }^{2,3}$, T. Di Salvo ${ }^{4,5}$, L. Burderi ${ }^{2}$, and G. Cocozza ${ }^{1}$ \\ 1 University of Rome 2, Via della Ricerca Scientifica 1, 00133 Roma, Italy \\ 2 INAF - Astronomical Observatory of Rome, Via Frascati 33, 00040 Monteporzio Catone, Italy \\ 3 Affiliated with ICRA \\ 4 Astronomical Institute "Anton Pannekoek", University of Amsterdam and Center for High-Energy Astrophysics, \\ Kruislaan 403, 1098 SJ Amsterdam, The Netherlands \\ 5 Dipartimento di Scienze Fisiche ed Astronomiche, Universita' di Palermo, via Archirafi 36, 90123 Palermo, Italy
}

Received 14 November 2003 / Accepted 10 March 2004

\begin{abstract}
We report on a spectral and timing analysis of BeppoSAX data of the $13.6 \mathrm{~s}$ period transient X-ray pulsar EXO 053109-6609.2. Observations were carried out in March 1997 and October 1998, catching the source during a high and a low emission state, respectively. Correspondingly, the X-ray luminosity is found at a level of $4.2 \times 10^{37} \mathrm{erg} \mathrm{s}^{-1}$ and $1.5 \times 10^{36} \mathrm{erg} \mathrm{s}^{-1}$ in the two states. In the high state the X-ray emission in the energy range $1-100 \mathrm{keV}$ is well fitted by an absorbed power-law with photon index $\Gamma \sim 1.7$ plus a blackbody component with a characteristic temperature of $\sim 3.5 \mathrm{keV}$. Moreover, we find evidence of iron emission at $\sim 6.8 \mathrm{keV}$, a typical feature in this class of sources but never seen before in the EXO 053109-6609.2 spectrum. In the low state an absorbed power-law with $\Gamma \sim 0.4$ is sufficient to fit the $1-10 \mathrm{keV}$ data. During BeppoSAX observations EXO 053109-6609.2 display variations of the pulse profile with the X-ray flux: it showed single peaked and double peaked profiles in the low and high state, respectively. Based on these two observations we infer a spin-up period derivative of $-(1.14 \pm 0.08) \times 10^{-10} \mathrm{ss}^{-1}$. By comparing these with other period measurements reported in the literature we find an alternating spin-up and spin-down behaviour that correlates well with the X-ray luminosity.
\end{abstract}

Key words. accretion, accretion disks - X-rays: stars - stars: neutron - X-rays: binaries

\section{Introduction}

EXO 053109-6609.2 is a High Mass X-ray Binary (HMXB) hosting a neutron star and a B-emission spectral type (Be) star (Haberl et al. 1995; McGowan \& Charles 2002). The system is in the Large Magellanic Cloud (LMC), 17' away from LMC X-4, another high mass X-ray binary pulsar (La Barbera et al. 2001). Be stars are characterised by high rotational velocities (up to $70 \%$ of their break-up velocity), and by episodes of equatorial mass loss which produce a temporary "decretion" disk around the star (Slettebak 1987; Okazaki \& Negueruela 2001). These X-ray binaries, in which a neutron star is orbiting in a relatively wide orbit with moderate eccentricity, are the most numerous among HMXBs. In the LMC, more than half of the confirmed HMXBs are variable or transient sources consisting of a neutron star with a Be companion (Haberl et al. 2003; Sasaki et al. 2000; Haberl \& Sasaki 2000).

Variable X-ray emission is often observed which likely results from the large variations in the Be wind density and relative velocities along the neutron star orbit, which in most cases may result in regular X-ray outbursts near the periastron (type I

Send offprint requests to: N. Rea, e-mail: rea@mporzio. astro. it outbursts). Alternatively, aperiodic outbursts occur which often last longer than the neutron star orbit. These are probably caused by matter ejection outflowing from the equatorial plane of the Be star (type II outbursts; Stella et al. 1986; Motch et al. 1991).

EXO 053109-6609.2 was discovered in 1983 deep EXOSAT exposures of the LMC X-4 region (Pakull et al. 1985; Pietsch et al. 1989). The luminosity was $\sim 6 \times 10^{36} \mathrm{erg} \mathrm{s}^{-1}$ (0.15-4 keV; assuming a distance of $50 \mathrm{kpc})$. Subsequently, the coded mask X-ray telescope SL2 XRT on board the shuttle Challenger between July and August 1985 detected a second outburst (Hanson et al. 1989), with a source luminosity of $\sim 1 \times$ $10^{37}$ erg s$^{-1}(2-10 \mathrm{keV})$.

The source was then monitored from June 1990 to July 1994 with the ROSAT PSPC (Haberl et al. 1995). Alternating high states $\left(L_{\mathrm{x}} \sim 10^{37} \mathrm{erg} \mathrm{s}^{-1}\right)$ and low states $\left(L_{\mathrm{x}} \sim 10^{36} \mathrm{erg} \mathrm{s}^{-1}\right)$ in the X-ray flux were discovered. Haberl et al. (1996) detected another outburst from March to May 1993 with an average luminosity $\sim 2.4 \times 10^{36} \mathrm{erg} \mathrm{s}^{-1}(0.1-2.4 \mathrm{keV})$.

Dennerl et al. (1996) reported on a ROSAT observation that took place in October 1991 and led to the detection of a spin period of $13.67133(5) \mathrm{s}$ with a period derivative 
of $(1.5 \pm 0.1) \times 10^{-8} \mathrm{ss}^{-1}$ (calculated during their observation). Haberl et al. (1995) proposed an orbital period of about 600-700 days and an orbital eccentricity of $e \sim 0.4-0.5$. Under the assumption that period changes are caused by Doppler shifts, Dennerl et al. (1996) corrected the proposed orbital period, finding an orbital solution with $P_{\text {orb }}=25.4$ days and an eccentricity of $e \sim 0.1$.

Timing analysis of the BeppoSAX data obtained in March 1997 when the system was in high-state revealed coherent pulsations at a period of 13.67590(8) s and two different period derivatives: a short-term period derivative, calculated during the 2 days of BeppoSAX observation, of $\dot{P}_{\text {loc }}=$ $(3.7 \pm 0.5) \times 10^{-9} \mathrm{ss}^{-1}$, and a secular period derivative, calculated from a comparison with a previous ROSAT measure of $\dot{P}_{\mathrm{sec}}=(3.67 \pm 0.05) \times 10^{-11} \mathrm{ss}^{-1}$ (Burderi et al. 1998).

The last observation of EXO 053109-6609.2 was a deep XMM-Newton observation of the LMC field of October 2000 (Haberl et al. 2003) in which EXO 053109-6609.2 was found to be the brightest source in the field $\left(7 \times 10^{-12} \mathrm{erg} \mathrm{cm}^{-2} \mathrm{~s}^{-1}\right.$ in $0.2-10 \mathrm{keV}$ range, corresponding to a luminosity of about $2.1 \times 10^{36} \mathrm{erg} \mathrm{s}^{-1}$ ).

Across different observations this source varied its intensity up to a factor of about 10 . Nevertheless, notwithstanding that most Be X-ray binary systems show a quiescent state, this source has not yet been detected in quiescence but only in a low-luminosity state with a minimum luminosity of $\sim 10^{35}-10^{36} \mathrm{erg} \mathrm{s}^{-1}$.

In this paper we report on spectral, spin period and pulse profile changes with the X-ray luminosity. We compare our results with previous findings.

\section{Observations}

BeppoSAX observed EXO 053109-6609.2 with its narrowfield instruments (NFI; Boella et al. 1997a) from 10:48:16 March 13, 1997 to 08:17:50 March 15, 1997 and from 22:39:47 October 20, 1998 to 08:05:31 October 22, 1998.

Spectral coverage of BeppoSAX extends over a wide energy range using four different instruments. In our analysis we use just two coaligned instruments: the Medium-Energy Concentrator Spectrometer (MECS) consisting of three units operating in the 1-10 keV range (Boella et al. 1997b) and the Phoswich Detector System (PDS) with four scintillation units operating in the range $10-200 \mathrm{keV}$ (Frontera et al. 1997). The High Pressure Gas Scintillator Proportional Counter (HPGSPC, 7-40 keV; Manzo et al. 1997) was not available. For the Low-Energy Concentrator Spectrometer (LECS, 0.1-4 keV; Parmar et al. 1997) in the low state observation there are not enough photons to be used, while for the high state the off-axis response matrix is not accurate enough (actually LECS data were not fully consistent with the MECS data in the overlapping range $\sim 1-5 \mathrm{keV}$ ). Both instruments used have a field of view of less than one degree but only MECS has imaging capabilities.

We extracted EXO 053109-6609.2 data from $4^{\prime}$ radius circular regions in the MECS field of view, centred on the maximum of the point spread function of the source. The data were background subtracted by extracting in the same field of view background photons from a similar circular region of $4^{\prime}$ radius, centred far enough from any sources present in the image and at the same off-axis position as EXO 053109-6609.2; the arrival times were then corrected for the barycentre of the solar system. Owing to the problems described above, we used only MECS and PDS data for the first observation: we could use the PDS data because the contaminating source LMC X-4 was in a low state during that observation (Burderi et al. 1998). During the second observation LMC X-4 was in a high state (Naik \& Paul 2004). In the PDS range, the relative fluxes between EXO 053109-6609.2 and LMC X-4 are: $F_{\mathrm{EXO}} / F_{\mathrm{LMC}} \sim 9$ for the first observation, and $F_{\mathrm{EXO}} / F_{\mathrm{LMC}} \sim 0.2$ for the second (the latter value was inferred extrapolating the EXO 053109-6609.2 low state spectrum in the PDS range). Because during the second observation LMC X-4 was greatly more luminous than EXO 053109-6609.2, and given that PDS does not have imaging capabilities, we only used MECS data in this case.

MECS spectra, accumulated from the same circular regions used for the event files, were re-binned with the BeppoSAX grouping file: mecs_5.grouping; PDS spectra were re-binned in order to have at least fifty photons for each bin. Obviously, both re-binnings are made such that minimum chi-square techniques could be reliably used in spectral fitting. Spectral analysis was performed using the energy range $1.65-10.5 \mathrm{keV}$ for the MECS and 15-60 keV for the PDS (data in the $60-100 \mathrm{keV}$ PDS range was bad), and taking into account the off-axis position of the source. We used off-axis response matrices made using the precise estimation of EXO 053109-6609.2 off axis. Note that by using on-axis spectral response matrices for a source that is off-axis, one wrongly estimates the source spectral parameters. In particular, the flux is underestimated while the spectrum appears softer than what it actually is (Mereghetti et al. 2000). Correspondingly, any extrapolation towards high-energies may be strongly underestimated. All energy bins for which the count rate was consistent with zero were not used in the spectral analysis.

\section{Timing analysis}

We searched for coherent pulsations of the source in both states. In the low state (second observation), in a period interval around the ROSAT period, the power spectrum and the epoch folding search show an evident signal at $v \sim 0.0732 \mathrm{~Hz}$ with a highly significant peak $(\sim 10 \sigma)$ corresponding to a period of $\sim 13.67 \mathrm{~s}$ (see Fig. 1). Hereafter, if not specified, all errors are at the $1 \sigma$ confidence level. To rely upon a more accurate period measurement, we used a phase fitting technique by fitting the phases of the modulation over four intervals of $\sim 21000 \mathrm{~s}$ each. With this method we found a spin period of $13.6705 \pm$ $0.0004 \mathrm{~s}$.

Timing analysis of the first observation has been performed giving a spin period value of $13.67590(8) \mathrm{s}$ (Burderi et al. 1998). Applying the same method to the March 1997 observation, we found a spin period of $13.67630 \pm 0.00003 \mathrm{~s}$ (consistent with the period found for the same observation in 


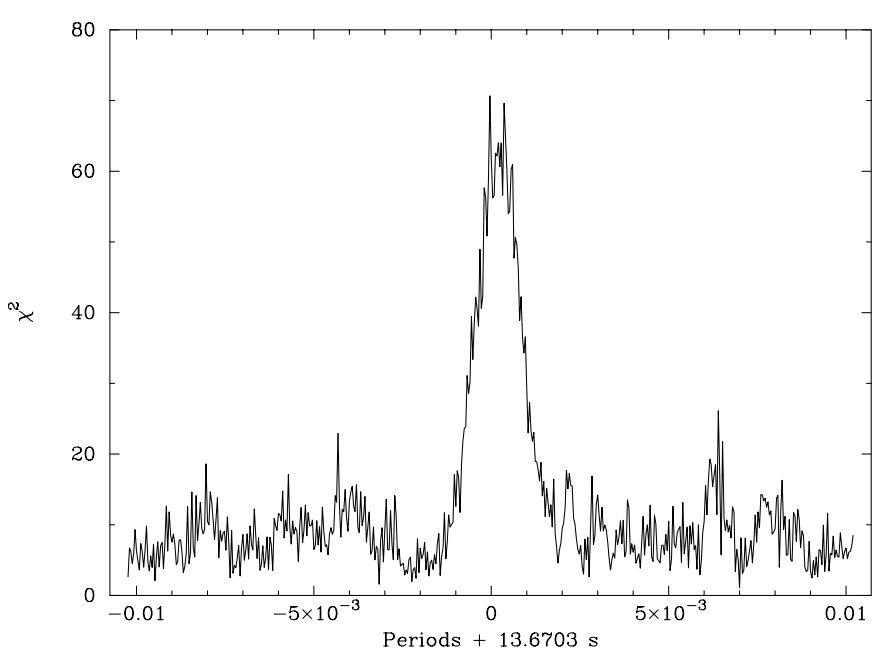

Fig. 1. Epoch folding search of the data from the low state observation of 1998.

Burderi et al. 1998) ${ }^{1}$. In the latter case, owing to higher statistics, we looked at the phase modulations over six intervals of $\sim 14000$ s: fitting the results with a polynomial function of the second order we found evidence for a quadratic component possibly related to the orbital motion of the X-ray pulsar (see Fig. 2). The local period derivative inferred fitting the phase residuals with a quadratic component is $\sim 7 \times 10^{-9} \mathrm{ss}^{-1}$.

The observation is unfortunately too short to look for an orbital solution. From the periods measured in these two BeppoSAX observations we inferred a $\dot{P}=-(1.14 \pm 0.08) \times$ $10^{-10} \mathrm{ss}^{-1}$.

The pulse profile obtained when the source was in the high state (March 1997) is dominated by the first harmonic. This predominance disappears at fainter fluxes (October 1998) where the fundamental is the strongest component (Fig. 3). Furthermore, the pulsed fraction (PF) in the MECS range changes from a value of $50 \pm 1 \%$ to $30 \pm 1 \%$ from high to low state, while in the PDS range only in the high state do we see the pulsation, and the PF is $40 \pm 4 \%$ (see Fig. 3). The low state PDS epoch folding at the period found in the MECS data did not show any modulation due to poor statistics (with an upper limit on the PF of $\sim 80 \%$ or $100 \%$ depending whether we estimate the PDS flux of LMC X-4 be 3.5 counts/s or 4.0 counts/s), correspondingly we cannot exclude an high-energy pulsation of the source in low state.

\section{Spectral analysis}

The EXO 053109-6609.2 high-state spectrum is well fitted by an absorbed blackbody plus power-law component and a Gaussian function (see Table 1 and Fig. 4). The inferred luminosity between $2-60 \mathrm{keV}$, assuming a distance of $50 \mathrm{kpc}$ (this is the distance known to the Large Magellanic Cloud), is $4.2 \times 10^{37} \mathrm{erg} \mathrm{s}^{-1}$. We tried to fit the spectrum with other

${ }^{1}$ Note that while the period in Burderi et al. (1998) is referred to the start of the observation, here we report the periods at the half time of the observation. Taking in account the local period derivative reported before, the two periods are consistent.

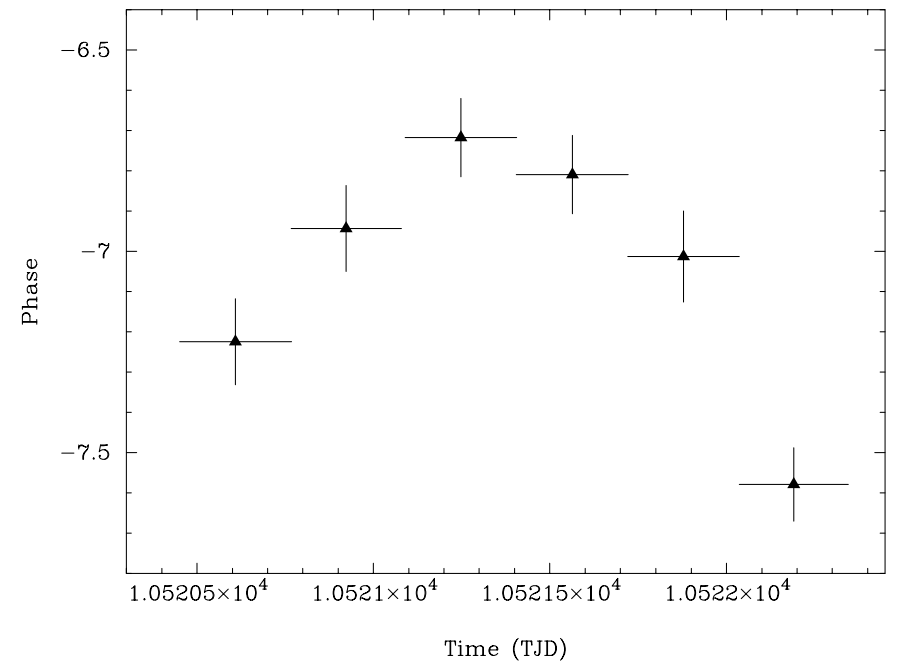

Fig. 2. Evidence of a quadratic component in the phase-fitting of the high-state observation (linear component relative to a period of $13.67 \mathrm{~s}$ was removed to better show the quadratic residuals).

Table 1. Spectral parameters of EXO 0531-66 in the high-state (absorbed blackbody plus a power-law and a Gaussian) and in low-state (absorbed power-law). Fluxes are unabsorbed and in units of $\times 10^{-11} \mathrm{erg} \mathrm{cm}^{-2} \mathrm{~s}^{-1}$ (see also Fig. 4); errors are $1 \sigma$ confidence level.

\begin{tabular}{lcc}
\hline \hline Spectral parameters & High state & Low state \\
\hline$N_{\mathrm{H}}\left(\times 10^{22} \mathrm{~cm}^{-2}\right)$ & $0.92 \pm 0.04$ & 0.92 (frozen) \\
Power-law PI & $1.75 \pm 0.08$ & $0.4 \pm 0.1$ \\
flux PL $(2-10 \mathrm{keV})$ & 2.0 & - \\
$k T_{\mathrm{bb}}(\mathrm{keV})$ & $3.53 \pm 0.3$ & - \\
Flux bb $(2-10 \mathrm{keV})$ & 1.9 & - \\
$E_{\mathrm{Fe}}(\mathrm{keV})$ & $6.8 \pm 0.1$ & - \\
$\sigma_{\mathrm{Fe}}(\mathrm{keV})$ & $0.4 \pm 0.1$ & - \\
$E Q W_{\mathrm{Fe}}(\mathrm{eV})$ & 275 & - \\
$\chi_{v}{ }^{2}$ & $1.18(169$ d.o.f. $)$ & $1.2(25$ d.o.f.) \\
Total flux $(2-10 \mathrm{keV})$ & 5.0 & 0.5 \\
Total flux $(2-60 \mathrm{keV})$ & 14.0 & - \\
\hline
\end{tabular}

models: two blackbody plus a power-law, a blackbody plus a cutoff power-law and a blackbody plus a high-energy cutoff model but all give worse results $\left(\chi_{v}^{2}>1.9\right)$. Fitting the spectrum with a blackbody plus a cutoff power-law we initially obtained a good chi-square value but the absorption was too small $\left(N_{\mathrm{H}}=(0.03 \pm 0.01) \times 10^{22}\right.$ atoms $\left./ \mathrm{cm}^{2}\right)$ for a system in the LMC. We then fixed the absorption value at $\sim 0.1 \times$ $10^{22}$ atoms $/ \mathrm{cm}^{2}$ but the chi-square becomes remarkably worse (this is the galactic absorption value calculated for the RA and Dec of EXO 053109-6609.2 with the $n h$ ftool; Dickey \& Lockman 1990). Adding a Gaussian line at $\sim 6.8 \mathrm{keV}$ (see Table 1) to the continuum model, the chi-square value is improved from 1.28 (172 d.o.f.) to 1.18 (169), giving an $\mathrm{F}$ test probability of $3.5 \times 10^{-4}$.

The low-state spectrum is modelled by an absorbed power-law (see Table 1 and Fig. 4) and the source luminosity in this state is $1.5 \times 10^{36} \mathrm{erg} \mathrm{s}^{-1}$ (for a distance of $50 \mathrm{kpc}$; in the $2-60 \mathrm{keV}$ energy range). We also tried to add a Gaussian 

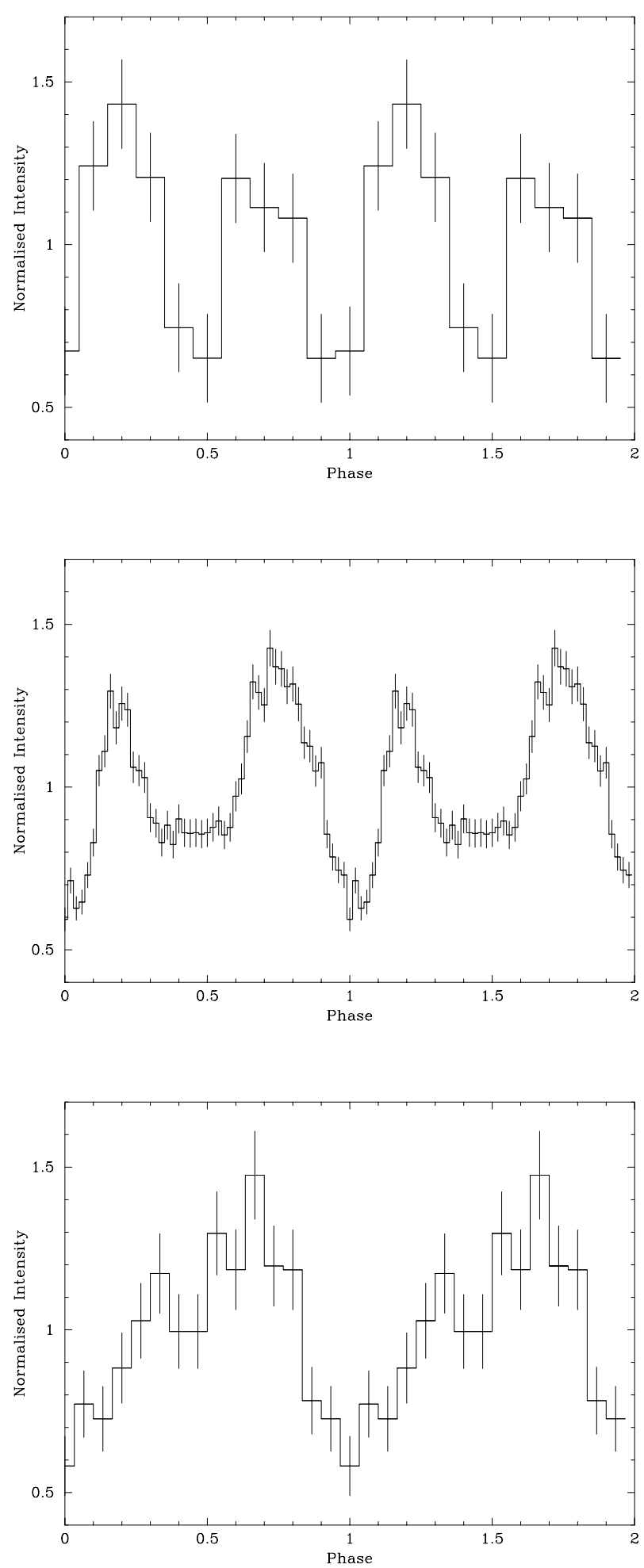

Fig. 3. First and second panels: pulse profile of March 1997 BeppoSAX observation of EXO 053109-6609.2 in PDS and MECS bands aligned in phase, respectively. Third panel: pulse profile of October 1998 BeppoSAX observation of EXO 053109-6609.2 in the MECS band, while the source was in the low-state (not phase aligned with previous observation) .

function at $\sim 6.8 \mathrm{keV}$ but it was no significative (confidence level $<1.5 \sigma)$.
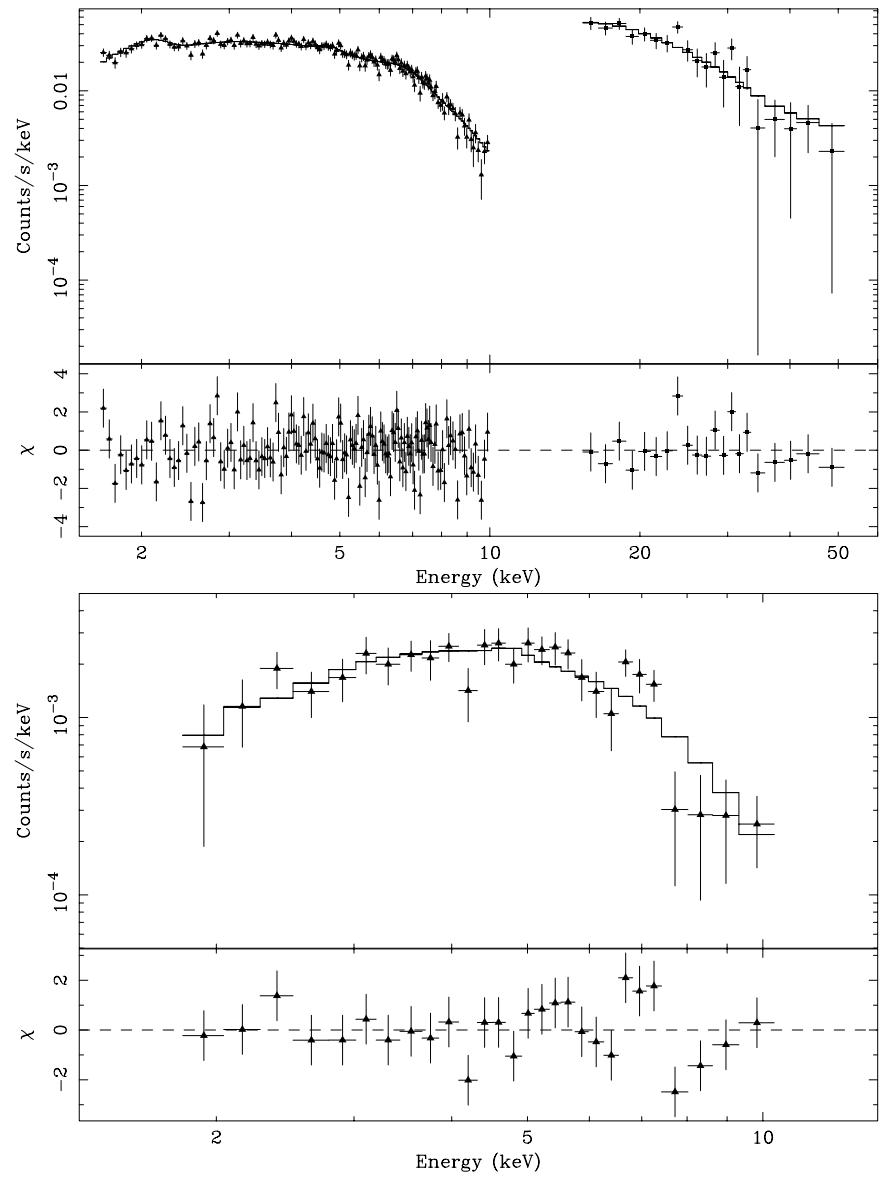

Fig. 4. BeppoSAX spectra of EXO 053109-6609.2 in high state (1997) and in low state (1998). See Table 1 for spectral parameters.

\section{Discussion}

During the outburst phase of Be X-ray binaries, accretion disks are expected to be present, and indeed, evidence for an accretion disk, based on a correlation between the observed flux and spin-up or spin-down rate, has been found for several sources (Bildsten et al. 1997; Okazaki \& Negueruela 2001). The EXO 053109-6609.2 spin period was detected four times: the first time by ROSAT (Dennerl et al. 1996), two times by BeppoSAX (Burderi et al. 1998, and this paper) and the fourth time by XMM-Newton satellite (Haberl et al. 2003). In Fig. 5 we plot the $0.1-2.4 \mathrm{keV}$ band luminosity versus period for these four observations; it is evident from the plot that periods and luminosities are directly correlated. Comparing the spin periods of all the observations of EXO 053109-6609.2 carried out since its discovery, we found that the source alternates between spin-up and spin-down (Fig. 5). The secular period derivative between the ROSAT observation (Haberl et al. $1995)$ and the first BeppoSAX observation is $(2.9 \pm 0.1) \times$ $10^{-11} \mathrm{ss}^{-1}$; comparing the two BeppoSAX observations we obtain $-(1.14 \pm 0.08) \times 10^{-10} \mathrm{ss}^{-1}$, and the $\dot{P}$ between the last BeppoSAX observation and the XMM observation (Haberl et al. 2003) is $-(3.7 \pm 0.1) \times 10^{-11} \mathrm{ss}^{-1}$ (period derivative errors are at the $90 \%$ confidence level). The secular spin period derivative is changed from a spin-down to a spin-up trend, 


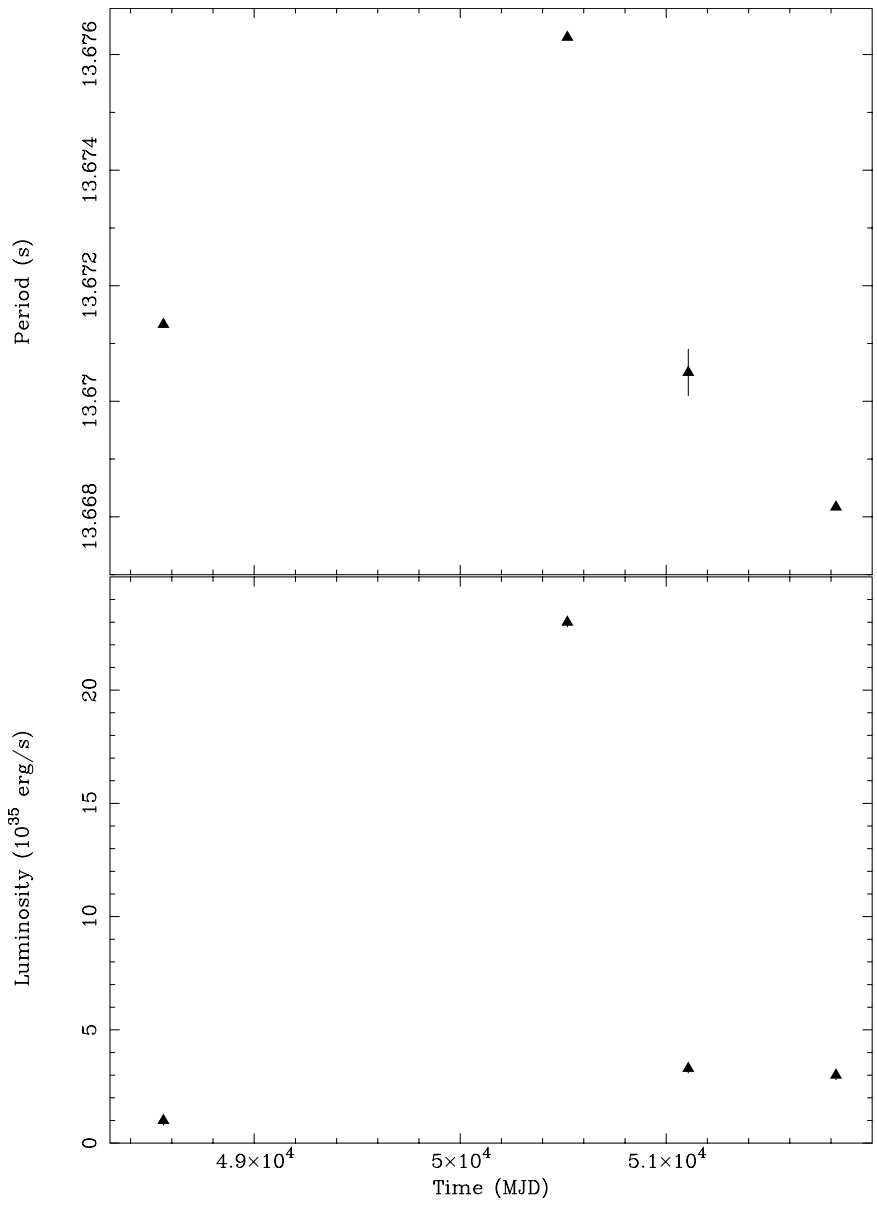

Fig. 5. Spin period and luminosity changes between ROSAT (Haberl et al. 1996), BeppoSAX (this paper) and XMM (Haberl et al. 2003) observations (all reported luminosities are extrapolated in $0.1-2.4 \mathrm{keV}$ band).

which has a clear correlation with the X-ray luminosity. We detected this change around 1997.

A double peaked pulse profile (Fig. 3, first and second panels) was observed when the source was in high-state, while a single peaked profile was found in the low-state (Fig. 3, third panel). This behaviour often has been seen in X-ray binary systems and has been ascribed to the transition of the source between a pencil beam and a pencil plus fan beam emission geometry, a behaviour which is correlated with the X-ray flux (Parmar et al. 1989a,b). Timing behaviour of this source is similar to what seen in other Be X-ray binaries, making the period derivative changes a peculiar characteristic of these systems.

The spectrum in the high-state of emission is well fitted by an absorbed blackbody plus a power law and a Gaussian line at $\sim 6.8 \mathrm{keV}$ (see Table 1 and Fig. 4 first panel). The $6.8 \mathrm{keV}$ emission line is probably due to $\mathrm{K}$-shell emission from highly ionized iron (probably in the He-like and/or H-like ionization stages). This is the first evidence of an iron emission line in this source; iron emission lines are a common feature in HMXBs, although their centroid energies are usually detected in the range 6.4-6.7 keV (e.g., Parmar et al. 1989a).
From measured temperature of $k T \sim 3.5 \mathrm{keV}$ we infer an emission radius for the blackbody component of $\sim 5.5 \mathrm{~km}$, about half a neutron star radius. The calculated blackbody radius is smaller than the neutron star radius, probably due to the fact that the thermal emission does not come from the whole neutron star surface but probably just from a small region around the polar caps. The energy flux of the two spectral components differs by about two orders of magnitude, $F_{\text {bb }} / F_{\text {power }}=1.728 \times 10^{-2}$. The blackbody component probably disappears while the system is in the low-state and the power-law photon index becomes flatter (see Table 1 and Fig. 4 second panel).

Acknowledgements. We thank T. Mineo for useful help with LECS response matrices and the anonymous referree for useful comments. This work started during an Astrophysical Laboratory of Prof. R. Buonanno in the University of Rome "Tor Vergata". This work is supported through ASI, CNR and Ministero dell'Università e Ricerca Scientifica e Tecnologica (MURST-COFIN) grants.

\section{References}

Bildsten, L., Chakrabarty, D., Chiu, J., et al. 1997, ApJS, 113, 367 Boella, G., Butler, R. C., Perola, G. C., et al. 1997a, A\&AS, 122, 299 Boella, G., Chiappetti, L., Conti, G., et al. 1997b, A\&AS, 122, 327 Burderi, L., Di Salvo, T., Robba, N. R., et al. 1998, ApJ, 498, 831 Dennerl, K., Haberl, F., \& Pietsch, W. 1996, MPE Report, 263, 131 Dickey, J. M., \& Lockman, F. J. 1990, ARA\&A, 28, 215

Frontera, F., Costa, E., dal Fiume, D., et al. 1997, A\&AS, 122, 357 Haberl, F., Denner, K., \& Pietsch, W. 1996, MPE Report, 263, 143

Haberl, F., Dennerl, K., \& Pietsch, W. 1995, A\&A, 302, L1

Haberl, F., Dennerl, K., \& Pietsch, W. 2003, A\&A, 406, 471

Haberl, F., \& Sasaki, M. 2000, A\&A, 359, 573

Hanson, C. G., Skinner, G. K., Eyles, C. J., \& Willmore, A. P. 1989 , MNRAS, 240, 1

La Barbera, A., Burderi, L., Di Salvo, T., Iaria, R., \& Robba, N. R. 2002, ApJ, 553, 375

Manzo, G., Giarrusso, S., Santangelo, A., et al. 1997, A\&AS, 122, 341

Mereghetti, S., Tiengo, A., Israel, G. L., \& Stella, L. 2000, A\&A, 354, 567

McGowan, K. E., \& Charles, P. A. 2002, MNRAS, 335, 941

Motch, C., Stella, L., Janot-Pacheco, E., \& Mouchet, M. 1991, ApJ, 369,490

Naik, S., \& Paul, B. 2004, ApJ, 600, 351

Okazaki, A. T., \& Negueruela, I. 2001, A\&A, 337, 161

Pakull, M., Brunner, H., Pietsch, W., et al. 1985, Space Sci. Rev., 40, 379

Parmar, A. N., White, N. E., \& Stella, L. 1989a, ApJ, 338, 373

Parmar, A. N., White, N. E., Stella, L., Izzo, C., \& Ferri, P. 1989b, ApJ, 338, 359

Parmar, A. N., Martin, D. D. E., Bavdaz, M., et al. 1997, A\&AS, 122, 309

Pietsch, W., Dennerl, K., \& Rosso, C. 1989, in Proc. 23rd ESLAB Symp., 296

Saski, M., Haberl, F., \& Pietsch, W. 2000, A\&AS, 143, 391

Slettebak, A. 1987, Physics of Be stars, Proc. IAU Symp., 92, Boulder, Colorado, 24

Stella, L., White, N., \& Rosner, R. 1986, ApJ, 308, 669 